\title{
THE S-MEASURE FOR ALGEBRAIC INTEGERS HAVING ALL THEIR CONJUGATES IN A SECTOR.
}

\author{
V. FLAMMANG
}

\begin{abstract}
Let $\alpha$ be a nonzero algebraic integer of degree $d$ whose all conjugates $\alpha_{1}=\alpha, \alpha_{2}, \ldots, \alpha_{d}$ lie in a sector $|\arg z| \leq \theta, 0<\theta \leq 90^{\circ}$. We define the S-measure of $\alpha$ by $\mathrm{S}(\alpha)=\sum_{i=1}^{d}\left|\alpha_{i}\right|$ and the absolute $\mathrm{S}$-measure of $\alpha$ by $\mathrm{s}(\alpha)=\mathrm{S}(\alpha) / d$. We compute the greatest lower bound $c(\theta)$ of $\mathrm{s}(\alpha)$ for $\alpha$ belonging to twelve subintervals of $(0, \theta)$. Among these subintervals, three are complete. These computations use the principle of explicit auxiliary functions and our recursive algorithm.
\end{abstract}

\section{Introduction}

Let $\alpha$ be a nonzero algebraic integer of degree $d$. We define the S-measure of $\alpha$ by:

$$
\mathrm{S}(\alpha)=\sum_{i=1}^{d}\left|\alpha_{i}\right| .
$$

The absolute $\mathrm{S}$-measure of $\alpha$ is the quantity $\mathrm{s}(\alpha)=\mathrm{S}(\alpha) / d$.

If $P$ is the minimal polynomial of $\alpha$, then $\mathrm{S}(P)=\mathrm{S}(\alpha)$ and $\mathrm{s}(P)=\mathrm{s}(\alpha)$.

The arithmetic-geometric inequality shows that $\mathrm{s}(\alpha) \geq 1$. Since $\alpha$ is a nonzero algebraic integer the quantity $\left|\alpha_{1} \ldots \alpha_{d}\right|$ is a positive integer, and therefore $\left|\alpha_{1} \ldots \alpha_{d}\right| \geq 1$. The equality holds if and only if $\alpha$ is a root of unity. Indeed, if $\mathrm{s}(\alpha)=1$, then $\frac{\sum_{i=1}^{d}\left|\alpha_{i}\right|}{d}=\prod_{i=1}^{d}\left|\alpha_{i}\right|^{1 / d}$. Thus, for $i=1, \ldots, d$, all the $\left|\alpha_{i}\right|$ are equal to each other and then equal to 1 . The theorem of Kronecker $[\mathrm{K}]$ implies that $\alpha$ is a root of unity.

In this paper, we consider an algebraic integer $\alpha$ whose all conjugates lie in a sector $|\arg z| \leq \theta$, $0<\theta \leq 90^{\circ}$.

Note that the case $\theta$ equals to 0 is well known. $\alpha$ is then a totally positive algebraic integer i.e., all its conjugates are positive real numbers. Thus, the S-measure of $\alpha$ is then the usual trace of $\alpha$ and was already studied by a lot of authors. For more details, see for example [S], [ABP], [AP1], [AP2], [LW], [DW], [F1].

Hence, we focus here our attention on $\theta>0$. We follow a work of M. Langevin [La] on the absolute Mahler measure $\Omega(\alpha)=\left(\prod_{i=1}^{d} \max \left(1,\left|\alpha_{i}\right|\right)\right)^{1 / d}$ of algebraic integers $\alpha$ having all their conjugates in a sector. He proved that there exists a function $c(\theta)$ on $\left[0,180^{\circ}\right)$, always $>1$, such that if $\alpha \neq 0$ is not a root of unity, whose conjugates all lie in $S_{\theta}=\{z|| \arg z \mid \leq \theta$, $\left.0 \leq \theta \leq 180^{\circ}\right\}$, then $\Omega(\alpha) \geq c(\theta)$. But his result was not numerical. G. Rhin and C. Smyth [RS] were the first to succeed in finding the exact value of $c(\theta)$ for $\theta$ in nine subintervals of $\left[0,120^{\circ}\right]$ and conjectured that $c(\theta)$ is a "staircase "function of $\theta$, which is constant except for finitely many left discontinuities in any closed subinterval of $\left[0,180^{\circ}\right)$. Their method used explicit auxiliary polynomials found heuristically. In 2004, thanks to Wu's algorithm [Wu], G. Rhin and $\mathrm{Q} . \mathrm{Wu}[\mathrm{RW}]$ gave the exact value of $c(\theta)$ for four new subintervals of $\left[0,140^{\circ}\right]$ and extended four existing subintervals. In 2013, the author and G. Rhin $[\mathrm{FR}]$ found for the first time a complete subinterval and a fourteenth subinterval. A complete subinterval is an interval on which the function $c(\theta)$ is constant, with jump discontinuities at each end. These improvements were due to our recursive algorithm. From there, we studied most of the usual measures for $\alpha$ having all its conjugates in a sector, in particular the usual trace [F2]. 
Here, we prove the following result

Theorem 1. There exist a left discontinuous, strictly positive, staircase function $g$ on $\left[0,90^{\circ}\right)$ and a positive, continuous, monotonically decreasing function $f$ on $\left[0,90^{\circ}\right)$ such that:

for $\theta \in\left[0,90^{\circ}\right)$, if $\alpha$ is a nonzero algebraic integer of degree $\geq 2$ whose conjugates all lie in $S_{\theta}=\left\{z|| \arg z \mid \leq \theta, 0 \leq \theta \leq 90^{\circ}\right\}$ then

$$
\mathrm{s}(\alpha) \geq \min (f(\theta), g(\theta))
$$

Moreover, the exact value of $c(\theta)=\inf _{|\arg \alpha| \leq \theta} \mathrm{s}(\alpha)$ is known on twelve subintervals of $\left[0,90^{\circ}\right)$.

These intervals are given in Table 1.

The function $g(\theta)$ is a decreasing staircase function having left discontinuities. The function $g(\theta)$ is the smallest value of $\mathrm{s}(\alpha)$ that could be found for $\alpha$ having all its conjugates in $|\arg z| \leq \theta$.

The function $f(\theta)$ is given by $f(\theta)=\max _{1 \leq i \leq 12}\left(f_{i}(\theta)\right)$, and the functions $f_{i}(\theta)$ are of the type:

$$
f_{i}(\theta)=\min _{z \in S_{\theta}}\left(|z|-\sum_{1 \leq j \leq J} c_{i j} \log \left|Q_{i j}(z)\right|\right)
$$

where the polynomials $Q_{i j}$ with integer coefficients and the positive numbers $c_{i j}$ are read off from Table 2.

Table 1 Intervals $\left[\theta_{i}, \theta_{i}{ }^{\prime}\right]$ where $c(\theta)$ is known exactly. Here $c(\theta)=c\left(\theta_{i}\right)=\mathrm{s}(P)$ for $\theta \in\left[\theta_{i}, \theta_{i}{ }^{\prime}\right]$. $P$ denotes the polynomial whose all conjugates lie in $S_{\theta_{i}}$. The angles are given in degrees.

$\begin{array}{lllll}i & c(\theta) & \theta_{i} & \theta_{i}{ }^{\prime} \quad P\end{array}$

$\begin{array}{ccccl}1 & 1.5 & 0 & 20.1 & x^{2}-3 x+1 \\ 2 & 1.428241 & 21.640384 & 26.408740 & x^{3}-4 x^{2}+5 x-1 \\ 3 & 1.395643 & 26.408740 & 29.86 & x^{4}-5 x^{3}+9 x^{2}-5 x+1 \\ 4 & 1.365660 & 32.569940 & 33.14 & x^{6}-7 x^{5}+21 x^{4}-32 x^{3}+25 x^{2}-8 x+1 \\ 5 & 1.280776 & 38.668282 & 40.65 & x^{4}-4 x^{3}+7 x^{2}-4 x+1 \\ 6 & 1.221499 & 47.941432 & 50.882970 & x^{6}-5 x^{5}+13 x^{4}-17 x^{3}+13 x^{2}-5 x+1 \\ 7 & 1.214392 & 50.882970 & 53.32 & x^{6}-5 x^{5}+13 x^{4}-18 x^{3}+15 x^{2}-6 x+1 \\ 8 & 1.196310 & 56.004692 & 57.66 & x^{8}-6 x^{7}+20 x^{6}-40 x^{5}+53 x^{4}-45 x^{3}+24 x^{2}-7 x+1 \\ 9 & 1.190591 & 59.066144 & 63.91 & x^{6}-4 x^{5}+10 x^{4}-13 x^{3}+11 x^{2}-4 x+1 \\ 10 & 1.144122 & 64.086354 & 72.86 & x^{4}-2 x^{3}+4 x^{3}-2 x+1 \\ 11 & 1.094450 & 76.795630 & 80.656154 & x^{4}-x^{3}+3 x^{2}-x+1 \\ 12 & 1.073092 & 80.656154 & 87.5 & x^{3}-x^{2}+2 x-1\end{array}$

\section{The explicit auxiliary functions}

Let $\alpha$ be an algebraic integer of degree $d$ all of which conjugates $\alpha_{1}=\alpha, \ldots, \alpha_{d}$ lie in $S_{\theta}=$ $\{z|| \arg z \mid \leq \theta\}$, with minimal polynomial $P$. The auxiliary functions $f_{i}, 1 \leq i \leq 12$, are of the following type:

$$
\forall z \in S_{\theta}, f(z)=|z|-\sum_{1 \leq j \leq J} c_{j} \log \left|Q_{j}(z)\right|,
$$

where the coefficients $c_{j}$ are positive real numbers and the polynomials $Q_{j}$ are nonzero in $\mathbb{Z}[z]$, not necessarily irreducible, but not divisible by $P$. The main point is to choose the numbers $c_{j}$ and the polynomials $Q_{j}$ in order to maximize the minimum $m$ of $f$ on $S_{\theta}$. 
If we have $\sum_{i=1}^{d} f\left(\alpha_{i}\right) \geq m d$ then

$$
S(\alpha)=\sum_{i=1}^{d}\left|\alpha_{i}\right| \geq m d+\sum_{j=1}^{J} c_{j} \log \left|\prod_{i=1}^{d} Q_{j}\left(\alpha_{i}\right)\right|
$$

Since $P$ does not divide any $Q_{j}, \prod_{i=1}^{d} Q_{j}\left(\alpha_{i}\right)$ is a nonzero integer because it is the resultant of $P$ and $Q_{j}$. Therefore, we have

$$
\mathrm{s}(\alpha) \geq m .
$$

The main difficulty in this procedure is to find a good list of polynomials $Q_{j}$ which gives a value of $m$ as large as possible. For this purpose, we link the auxiliary function to the integer transfinite diameter in order to find our polynomials by the recursive algorithm.

\section{Auxiliary functions and integer transfinite diameter}

In this section, we will need the following definition:

Let $K$ be a compact subset of $\mathbb{C}$. We define the integer transfinite diameter of $K$ by

$$
\begin{aligned}
& t_{\mathbb{Z}}(K)=\liminf _{n \geq 1} \quad \inf _{P \in \mathbb{Z}[z]}|P|_{\infty, K}^{\frac{1}{n}}, \\
& n \rightarrow \infty \quad \operatorname{deg}(P)=n
\end{aligned}
$$

where $|P|_{\infty, K}=\sup _{z \in K}|P(z)|$. If $\varphi$ is a positive function defined on $K$, the $\varphi$-integer transfinite diameter of $K$ is defined as

$$
\begin{array}{ccc}
t_{\mathbb{Z}, \varphi}(K)= & \liminf \quad \inf ^{n \geq 1} \quad \sup \quad\left(|P(z)|^{\frac{1}{n}} \varphi(z)\right) . \\
& n \rightarrow \infty & \operatorname{deg}(P)=n
\end{array}
$$

This weighted version of the integer transfinite diameter was introduced by F. Amoroso [A]. It is an important tool in the study of rational approximations of logarithms of rational numbers. Inside the auxiliary function (2.1), we replace the numbers $c_{j}$ by rational numbers $a_{j} / q$ where $q$ is a common denominator of the $c_{j}$ for $1 \leq j \leq J$. Then we can write:

$$
\text { for } z \in S_{\theta}, f(z)=|z|-\frac{t}{r} \log |Q(z)| \geq m
$$

where $Q=\prod_{j=1}^{J} Q_{j}^{a_{j}} \in \mathbb{Z}[z]$ is of degree $r=\sum_{i=1}^{J} a_{j} \operatorname{deg} Q_{j}$ and $t=\sum_{j=1}^{J} c_{j} \operatorname{deg} Q_{j}$. We want to get a function whose minimum $m$ in the sector $S_{\theta}$ is as large as possible. Thus we search for a polynomial $Q \in \mathbb{Z}[z]$ such that

$$
\sup _{z \in S_{\theta}}|Q(z)|^{t / r}\left(e^{|z|}\right)^{-1} \leq e^{-m}
$$

If we suppose that $t$ is fixed, it is clear that we need an effective upper bound for the quantity

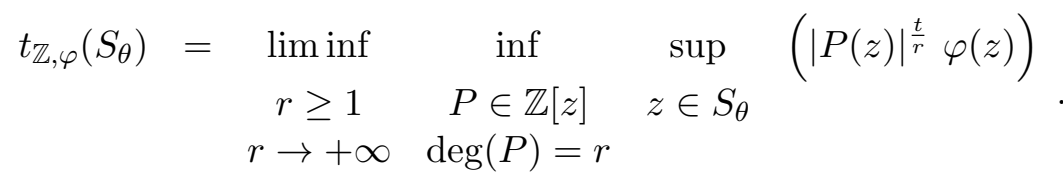

where we use the weight $\varphi(z)=\left(e^{|z|}\right)^{-1}$. 


\section{Construction of the auxiliary functions}

The polynomials involved in the auxiliary functions are found by our recursive algorithm developed in [F3] from Wu's algorithm [Wu]. It replaces a heuristic search for suitable polynomials by a systematic inductive search. Suppose that we have already found a list $Q_{1}, Q_{2}, \ldots, Q_{J}$ of suitable polynomials. Then we use semi-infinite linear programming (introduced into number theory by C.J. Smyth $[\mathrm{S}]$ ) to optimize $f$ for this set of polynomials (i.e., to get the greatest possible $m$ ). We obtain the real positive numbers $c_{1}, c_{2}, \ldots, c_{J}$ and then $f$ in the form (3.1) as above. The function $f$ is invariant under complex conjugation so we can limit ourselves to the sector $S_{\theta}^{\prime}=\{z \in \mathbb{C}$ such that $0 \leq \arg z \leq \theta\}$. Since the function $f$ is harmonic in $S_{\theta}^{\prime}$ outside the union of arbitrarily small disks around the roots of the polynomials $Q_{j}$, the minimum is taken on the boundary of $S_{\theta}^{\prime}$. Thus, it is sufficient to consider the minimum of $f$ on the half line $R_{\theta}=\left\{z=s e^{i \theta}, s \geq 0\right\}$.

The auxiliary function on the half line is:

$$
f(z)=s-\sum_{1 \leq j \leq J} c_{j} \log \left|Q_{j}(z)\right|
$$

Thus, by our algorithm, we seek a polynomial $R(z)=\sum_{l=0}^{k} a_{l} z^{l} \in \mathbb{Z}[z]$, where $k$ is varying from 4 to 15 successively, such that

$$
\sup _{s \geq 0}\left|Q\left(s e^{i \theta}\right) R\left(s e^{i \theta}\right)\right|^{\frac{t}{r+k}}\left(e^{s}\right)^{-1} \leq e^{-m}
$$

i.e., such that

$$
\sup _{s \geq 0}\left|Q\left(s e^{i \theta}\right) R\left(s e^{i \theta}\right)\right|\left(e^{s}\right)^{-\frac{r+k}{t}}
$$

is as small as possible.

But, here, $R\left(s e^{i \theta}\right)$ is not a linear forms in the unknown coefficients $a_{i}$ of $R$. We replace them by their real parts and their imaginary parts. Then, we get the following real linear forms

$$
\left|Q\left(s_{n} e^{i \theta}\right)\right| \operatorname{Re}\left(R\left(s_{n} e^{i \theta}\right)\right)\left(e^{s_{n}}\right)^{-\frac{r+k}{t}} \text { and }\left|Q\left(s_{n} e^{i \theta}\right)\right| \operatorname{Im}\left(R\left(s_{n} e^{i \theta}\right)\right)\left(e^{s_{n}}\right)^{-\frac{r+k}{t}} .
$$

The $s_{n}$ are suitable points in $(0, \infty)$, including the points where $f$ has its least local minima. All these linear forms define a real lattice on $\mathbb{Z}^{k+1}$. We use algorithm LLL to obtain a small vector in this lattice.

Then, we get a polynomial $R$ whose factors $R_{j}$ are good candidates to enlarge the set of polynomials $\left(Q_{1}, Q_{2}, \ldots, Q_{J}\right)$. We only keep the polynomials $R_{j}$ which have a nonzero coefficient $c_{j}$ in the newly optimized auxiliary function $f$. After optimization, some previous polynomials $Q_{j}$ may have a zero coefficient $c_{j}$ and so are removed. 
Table 2: The auxiliary functions $f_{i}, 1 \leq i \leq 12$.

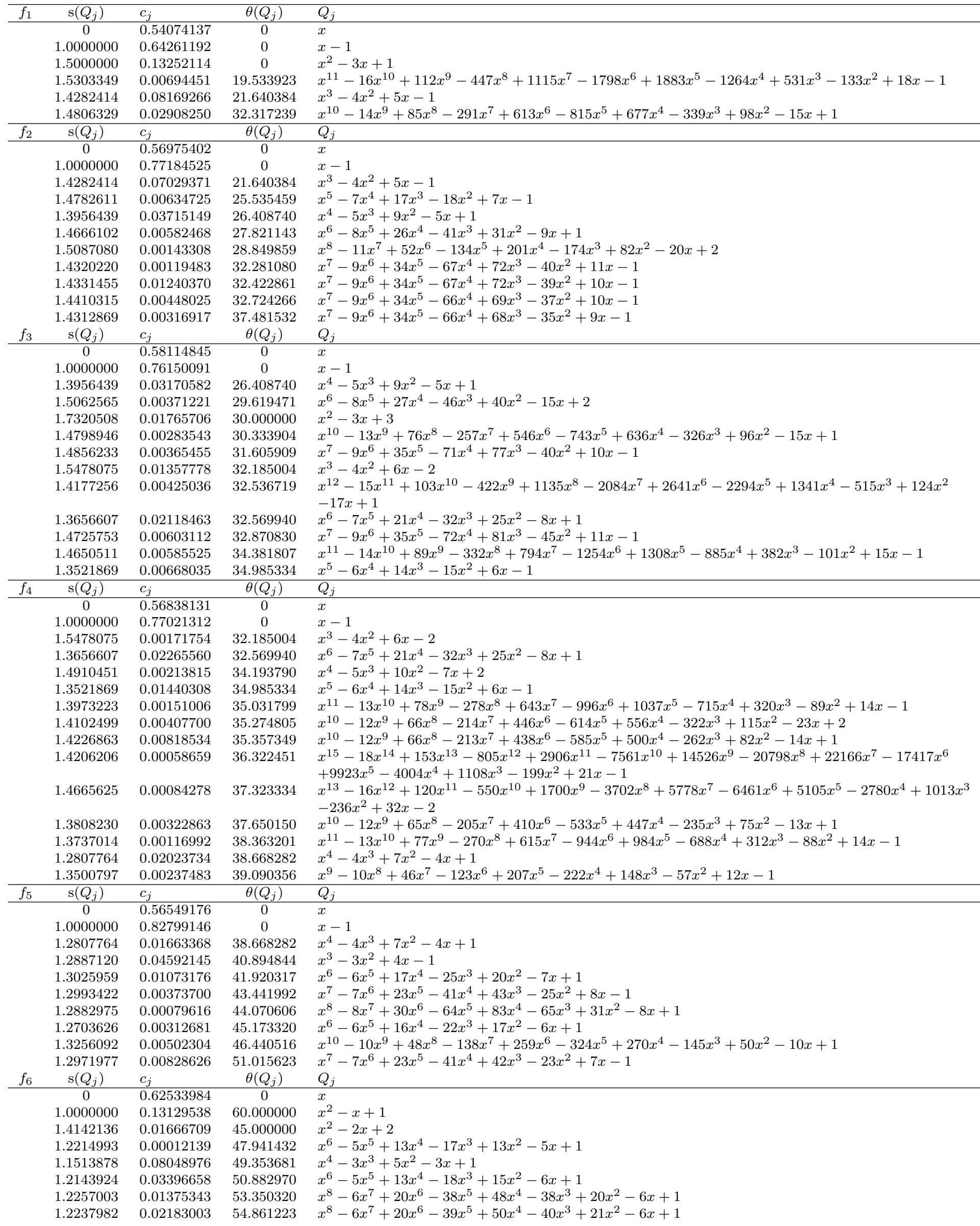




\begin{tabular}{|c|c|c|c|c|}
\hline$f_{7}$ & $\mathrm{~s}\left(Q_{j}\right)$ & $c_{j}$ & $\theta\left(Q_{j}\right)$ & $Q_{j}$ \\
\hline & 0 & 0.64156873 & 0 & $x$ \\
\hline & 1.4142136 & 0.02560466 & 45.000000 & $x^{2}-2 x+2$ \\
\hline & 1.1513878 & 0.08849685 & 49.353681 & $x^{4}-3 x^{3}+5 x^{2}-3 x+1$ \\
\hline & 1.2143924 & 0.02688682 & 50.882970 & $x^{6}-5 x^{5}+13 x^{4}-18 x^{3}+15 x^{2}-6 x+1$ \\
\hline & 1.2257003 & 0.01486371 & 53.350320 & $x^{8}-6 x^{7}+20 x^{6}-38 x^{5}+48 x^{4}-38 x^{3}+20 x^{2}-6 x+1$ \\
\hline & 1.2237982 & 0.01372826 & 54.861223 & $x^{8}-6 x^{7}+20 x^{6}-39 x^{5}+50 x^{4}-40 x^{3}+21 x^{2}-6 x+1$ \\
\hline & 1.0000000 & 0.12411259 & 60.000000 & $x^{2}-x+1$ \\
\hline \multirow[t]{7}{*}{$f_{8}$} & $\mathrm{~s}\left(Q_{j}\right)$ & $c_{j}$ & $\theta\left(Q_{j}\right)$ & $Q_{j}$ \\
\hline & 0 & 0.66258051 & 0 & $x$ \\
\hline & 1.1513878 & 0.08777864 & 49.353681 & $x^{4}-3 x^{3}+5 x^{2}-3 x+1$ \\
\hline & 1.1963101 & 0.00140181 & 56.004692 & $x^{8}-6 x^{7}+20 x^{6}-40 x^{5}+53 x^{4}-45 x^{3}+24 x^{2}-7 x+1$ \\
\hline & 1.1598550 & 0.01093296 & 59.015770 & $x^{3}-2 x^{2}+3 x-1$ \\
\hline & 1.1905913 & 0.02037738 & 59.066144 & $x^{6}-4 x^{5}+10 x^{4}-13 x^{3}+11 x^{2}-4 x+1$ \\
\hline & 1.0000000 & 0.19173198 & 60.000000 & $x^{2}-x+1$ \\
\hline \multirow[t]{7}{*}{$f_{9}$} & $\mathrm{~s}\left(Q_{j}\right)$ & $c_{j}$ & $\theta\left(Q_{j}\right)$ & $Q_{j}$ \\
\hline & 0 & 0.69106953 & 0 & $x$ \\
\hline & 1.1905913 & 0.00047744 & 59.066144 & $x^{6}-4 x^{5}+10 x^{4}-13 x^{3}+11 x^{2}-4 x+1$ \\
\hline & 1.0000000 & 0.20669082 & 60.000000 & $x^{2}-x+1$ \\
\hline & 1.2329260 & 0.00070272 & 63.571914 & $x^{7}-4 x^{6}+12 x^{5}-19 x^{4}+23 x^{3}-15 x^{2}+7 x-1$ \\
\hline & 1.1441228 & 0.06530631 & 64.086354 & $x^{4}-2 x^{3}+4 x^{2}-2 x+1$ \\
\hline & 1.2104994 & 0.02478898 & 64.985950 & $x^{7}-4 x^{6}+12 x^{5}-19 x^{4}+22 x^{3}-14 x^{2}+5 x-1$ \\
\hline \multirow[t]{8}{*}{$f_{10}$} & $\mathrm{~s}\left(Q_{j}\right)$ & $c_{j}$ & $\theta\left(Q_{j}\right)$ & $Q_{j}$ \\
\hline & 0 & 0.65189622 & 0 & $x$ \\
\hline & 1.0000000 & 0.20329094 & 60.000000 & $x^{2}-x+1$ \\
\hline & 1.1441228 & 0.05925279 & 64.086354 & $x^{4}-2 x^{3}+4 x^{2}-2 x+1$ \\
\hline & 1.4142136 & 0.01542631 & 69.295189 & $x^{2}-x+2$ \\
\hline & 1.1660867 & 0.01043488 & 73.001452 & $x^{8}-3 x^{7}+10 x^{6}-14 x^{5}+20 x^{4}-14 x^{3}+10 x^{2}-3 x+1$ \\
\hline & 1.1710692 & 0.02295000 & 74.639018 & $x^{8}-3 x^{7}+10 x^{6}-15 x^{5}+22 x^{4}-16 x^{3}+11 x^{2}-3 x+1$ \\
\hline & 1.1435156 & 0.00095069 & 77.579788 & $x^{6}-2 x^{5}+6 x^{4}-5 x^{3}+6 x^{2}-2 x+1$ \\
\hline \multirow[t]{6}{*}{$f_{11}$} & $\mathrm{~s}\left(Q_{j}\right)$ & $c_{j}$ & $\theta\left(Q_{j}\right)$ & $Q_{j}$ \\
\hline & 0 & 0.67127297 & 0 & $x$ \\
\hline & 1.0944505 & 0.029235734 & 76.795630 & $x^{4}-x^{3}+3 x^{2}-x+1$ \\
\hline & 1.1307279 & 0.01817266 & 84.841604 & $x^{6}-x^{5}+5 x^{4}-3 x^{3}+5 x^{2}-x+1$ \\
\hline & 1.1476672 & 0.02486566 & 86.221414 & $x^{6}-x^{5}+5 x^{4}-3 x^{3}+6 x^{2}-x+1$ \\
\hline & 1.0000000 & 0.22968256 & 90.000000 & $x^{2}+1$ \\
\hline \multirow[t]{5}{*}{$f_{12}$} & $\mathrm{~s}\left(Q_{j}\right)$ & $c_{j}$ & $\theta\left(Q_{j}\right)$ & $Q_{j}$ \\
\hline & 0 & 0.74200412 & 0 & $x$ \\
\hline & 1.0944505 & 0.08810863 & 76.795630 & $x^{4}-x^{3}+3 x^{2}-x+1$ \\
\hline & 1.0730921 & 0.002505677 & 80.656154 & $x^{3}-x^{2}+2 x-1$ \\
\hline & 1.0000000 & 0.14047981 & 90.000000 & $x^{2}+1$ \\
\hline
\end{tabular}

\section{References}

[A] F. Amoroso. f-transfinite diameter and number-theoretic applications, Ann. Inst. Fourier, Grenoble, 43 (1993), 11791198.

[ABP] J. Aguirre, M. Bilbao, J. C. Peral. The trace of totally positive algebraic integers, Math. Comp.75 (2006), no 253, 385-393.

[AP1] J. Aguirre and J.C. Peral. The integer Chebyshev constant of Farey intervals, Publ. Mat. 2007, Proceedings of the Primeras Jornadas de Teoría de Números, 11-27.

[AP2] J. Aguirre and J.C. Peral. The trace problem for totally positive algebraic integers. With an appendix by Jean-Pierre Serre, London Math. Soc. Lecture Note Ser., 352, Number theory and polynomials, 1-19, Cambridge Univ. Press, Cambridge, 2008.

[DW] X. Dong and Q. Wu. The absolute trace of totally positive reciprocal algebraic integers, J. Number Theory 170 (2017), $66-74$.

[F1] V.Flammang. Une nouvelle minoration pour la trace absolue des entiers algébriques totalement positifs available on hal-01346165v1, (2016)

[F2] V.Flammang. On the absolute trace of polynomials having all zeros in a sector, Experiment. Math. 17 (2008), no. 4, $443-450$.

[F3] V.Flammang. Trace of totally positive algebraic integers and integer transfinite diameter, Math. Comp. 78 (2009), no. $266,1119-1125$.

[FR] V. Flammang, G. Rhin. On the absolute Mahler measure of polynomials having all zeros in a sector. III, Math. Comp. 84 (2015), no. 296, 2927-2938.

[K] L. Kronecker. Zwei Sätze über Gleichugen mit ganzzahligen Koeffizienten, J. reine angew. Math.53 (1857), 173-175.

[La] M. Langevin. Minorations de la maison et de la mesure de Mahler de certains entiers algébriques, C. R. Acad. Sci. Paris, 303 (1986), 523-526. 
[LW] Y. Liang and Q. Wu. The trace problem for totally positive algebraic integers, J. Aust. Math. Soc. 90 (2011), no. 3, $341-354$

[RS] G. Rhin, C.J. Smyth. On the absolute Mahler measure of polynomials having all zeros in a sector, Math. Comp. 64 (209) (1995), 295-304.

[RW] G. Rhin, Q. Wu. On the absolute Mahler measure of polynomials having all zeros in a sector II, Math. Comp. $\mathbf{7 4}$ (249)(2004), 383-388.

[S] C.J. Smyth. The mean value of totally real algebraic numbers, Math. Comp. 42 (1984), 663-681.

[Wu] Q. Wu. On the linear independence measure of logarithms of rational numbers, Math. Comp. 72 (2003),901-911.

UMR CNRS 7502. IECL, Université de Lorraine, site de Metz,

Département de Mathématiques, UFR MIM,

3 rue Augustin Fresnel BP 4511257073 Metz cedex 3 E-mail address : valerie.flammang@univ-lorraine.fr 